TITLE:

\title{
Near-field scanning optical microscopic transient lens for carrier dynamics study in InGaN/GaN
}

\author{
$\operatorname{AUTHOR}(\mathrm{S})$ :
}

Okamoto, K; Scherer, A; Kawakami, Y

\section{CITATION:}

Okamoto, K ...[et al]. Near-field scanning optical microscopic transient lens for carrier dynamics study in InGaN/GaN. APPLIED PHYSICS LETTERS 2005, 87(16): 161104.

\section{ISSUE DATE:}

2005-10-17

URL:

http://hdl.handle.net/2433/50149

\section{RIGHT:}

Copyright 2005 American Institute of Physics. This article may be downloaded for personal use only. Any other use requires prior permission of the author and the American Institute of Physics. 


\title{
Near-field scanning optical microscopic transient lens for carrier dynamics study in InGaN/GaN
}

\author{
Koichi Okamoto ${ }^{\text {a) }}$ and Axel Scherer \\ Department of Physics, California Institute of Technology, Pasadena, California 91125 \\ Yoichi Kawakami \\ Department of Electronic Science and Engineering, Kyoto University, Kyoto 615-8510, Japan
}

(Received 29 June 2005; accepted 25 August 2005; published online 11 October 2005)

\begin{abstract}
Time-resolved microscopic transient lens (TR-M-TL) and near-field scanning optical microscopic transient lens (NSOM-TL) were performed to reveal temporal and spatial behavior of carrier dynamics in InGaN/GaN quantum wells. The carrier and thermal dynamics were observed through the time profile of the TR-M-TL signal. Also, NSOM-photoluminescence and NSOM-TL images were observed at the same time. By comparing these two images, both radiative and nonradiative recombination centers in InGaN active layer were unambiguously discriminated with submicrometer scale. Such nonradiative carrier dynamics has been difficult to observe by conventional techniques in spite of its importance. () 2005 American Institute of Physics.
\end{abstract}

[DOI: $10.1063 / 1.2105999$ ]

InGaN quantum well (QW)-based light-emitting diodes (LEDs) are expected to eventually replace more traditional fluorescent tubes as illumination sources. ${ }^{1,2}$ In order to improve the efficiency of light emission from InGaN QW, we have to elucidate and understand the carrier dynamics and light emission mechanics. There are a lot of reports on the spatial resolved studies for $\mathrm{InGaN}$ by cathodoluminescence, ${ }^{3-5}$ near-field scanning optical microscopy (NSOM) ${ }^{6-12}$ or scanning confocal microscopy. ${ }^{13,14}$ By these techniques, the radiative carrier recombination process has been observed with submicrometer scale. However, these techniques cannot observe the nonradiative processes of carriers in spite of its importance. Recently, we have reported the time-resolved measurement with NSOM and have succeeded in the direct observation of the carrier diffusion and localization process. ${ }^{15,16}$ However, direct observation of the nonradiative carrier recombination process has still been very difficult.

Recently, we succeeded in the direct observation of the carrier diffusion, nonradiative recombination, and thermarization processes by using the transient grating (TG) technique. ${ }^{17,18}$ Also we reported the spatial resolved measurements of carrier and thermal dynamics by the transient lens (TL) technique. ${ }^{19,20}$ The TG and TL techniques measure the third-order nonlinear response induced in the materials by the excitation laser. In this letter, the TL technique is developed to the time-resolved measurement (TR-M-TL) and near-field scanning optical microscopic measurement (NSOM-TL), and applied to the carrier dynamics study in $\mathrm{InGaN} / \mathrm{GaN}$ QWs.

The experimental setup of the TR-M-TL is shown in Fig. 1(a). A frequency-tripled $\mathrm{Nd}$ : yttrium-aluminum-garnet laser $(355 \mathrm{~nm})$ and a continuous wave (cw)-He-Ne laser $(633 \mathrm{~nm})$ were used as pump and probe beams, respectively. The pulse width, power and repetition rate of the pump beam are $10 \mathrm{~ns}$, $0.3 \mathrm{~mJ} /$ pulse and $3 \mathrm{~Hz}$, respectively. Both pump and probe beams were focused by an objective lens $(\times 40)$ on the

a) Author to whom correspondence should be addressed; electronic mail: kokamoto@caltech.edu sample. The diameter $(\phi)$ of the focused laser spot is $\phi=3 \mu \mathrm{m}$. Focusing or defocusing of the probe beam by the transient lens effect was detected with a pinhole, a glass filter, and a photomultiplier.

The experimental setup of the NSOM-TL is shown in Fig. 1(a). A similar setup has been reported for the photothermal measurement of the NSOM. ${ }^{21}$ We used a Twin-SNOM system (OMICRON) with a time-modulated $(400 \mathrm{~Hz}) \mathrm{InGaN}$ laser $(405 \mathrm{~nm})$ and a cw-AlGaAs laser $(830 \mathrm{~nm})$ as pump and probe beams, respectively. Both beams were coupled into a metal-coated optical fiber tip. The aperture size at the end of the tip was $200 \mathrm{~nm}$, and the pump and probe beam power levels, before coupling into the optical fiber, were 5 and $1 \mathrm{~mW}$, respectively. The NSOM-photoluminescence (NSOM-PL) signal and the NSOM-TL signal were separated by a dichroic mirror and colored glass filter and detected with a photomultiplier and a highly sensitive (fW) InGaAs photodetector with a lock-in amplifier system, respectively. The distance between the tip and the sample surface is con-
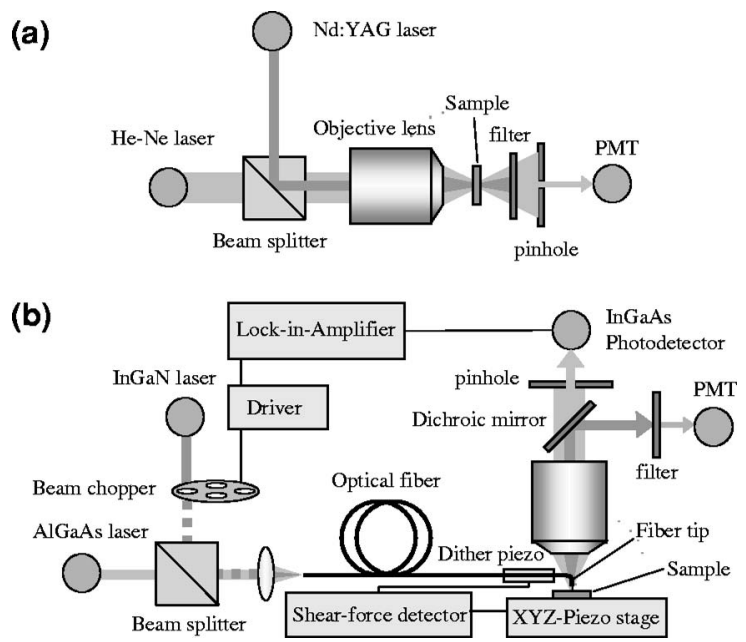

FIG. 1. (a) Experimental setup for (a) time-resolved microtransient lens (TR-M-TL) and (b) near-field scanning optical microscopic transient lens (NSOM-TL). 


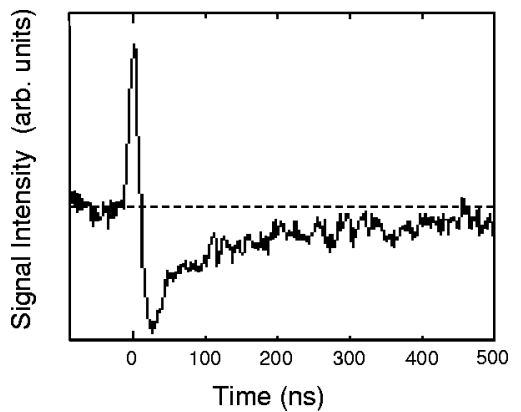

FIG. 2. Typical time profile of the TR-M-TL signals taken for the $\mathrm{InGaN} / \mathrm{GaN}$

trolled to $\sim 10 \mathrm{~nm}$ by shear-force detection. The sample stage was scanned in an $x-y$ direction by a piezo actuator.

The InGaN/GaN/AlGaN-based LED wafer, grown by a metalorganic chemical vapor deposition, was purchased from AXT Inc. The sample is composed of a sapphire substrate, a GaN buffer, an $n$-GaN, InGaN/GaN 3 QW active layers, a $p$-AlGaN, and a $p$-GaN cap layer. This wafer has light emission at the green wavelength region (peak: $525 \mathrm{~nm}$ ).

Figure 2(a) shows the typical time profile of the TRM-TL signals. The observed signal has two components, namely a spike-like positive component and a slowly decaying negative component. The positive and negative signals were produced by the focused and defocused probe beam by the TL generated in the sample. By solving ABCD law, it was found that these signal intensities are proportional to the refractive index change $(\delta n)$ induced by excitation. ${ }^{19}$ The induced carrier density change $(\delta N)$ and subsequently induced temperature change $(\delta T)$ by nonradiative recombination can contribute to the TL signal. Time $(t)$ and space $(x)$ dependent $\delta n$ is given by

$$
\delta n(x, t)=\left(\frac{\partial n}{\partial N}\right) \delta N(x, t)+\left(\frac{\partial n}{\partial T}\right) \delta T(x, t) .
$$

It was already known that the sign of $(\partial n / \partial N)$ is positive ${ }^{22}$ while the sign of $(\partial n / \partial T)$ is negative. ${ }^{23}$ From these relationships, we assigned the fast and slow components to the convex lens effect of the $\delta N$ and the concave lens effect of the $\delta T$, respectively. $\delta N(x, t)$ and $\delta T(x, t)$ are given by the following rate equations:

$$
\begin{aligned}
& \frac{d \delta N(x, t)}{d t}=D \frac{\partial^{2} \delta N(x, t)}{\partial t^{2}}-\left(\frac{1}{\tau_{\text {rad }}}+\frac{1}{\tau_{\text {nonrad }}}\right) \delta N(x, t), \\
& \frac{d \delta T(x, t)}{d t}=\frac{1}{\tau_{\text {nonrad }}} \frac{Q \delta N(x, t)}{\rho C p}+D_{\text {th }} \frac{\partial^{2} \delta T(x, t)}{\partial t^{2}},
\end{aligned}
$$

where $\tau_{\text {rad }}$ and $\tau_{\text {nonad }}$ are the radiative and nonradiative recombination lifetimes of carriers, $D$ and $D_{\text {th }}$ are the diffusion coefficient of carriers and the thermal diffusivity of the material, $Q, \rho$ and $C_{p}$ are the generated heat from unit density of carriers, material density and the heat capacity of material, respectively.

Since these simultaneous equations are difficult to resolve analytically, we solved them by numerical calculation. Figure 3(a) depicts the time development of the spatial distribution of the TL with $D=0.5 \mathrm{~cm}^{2} \mathrm{~s}^{-1}, D_{\text {th }}=1.0 \mathrm{~cm}^{2} \mathrm{~s}^{-1}$, and $\tau_{\mathrm{PL}}=20 \mathrm{~ns}$ (where $1 / \tau_{\mathrm{PL}}=1 / \tau_{\text {rad }}+1 / \tau_{\text {nonrad }}$ ). These values were obtained by the previous experiments. ${ }^{17-19}$ We used adequate proportional constant to each term and assumed a Downloaded 05 Mar 2008 to 130.54 .110.22. Redistribution subject
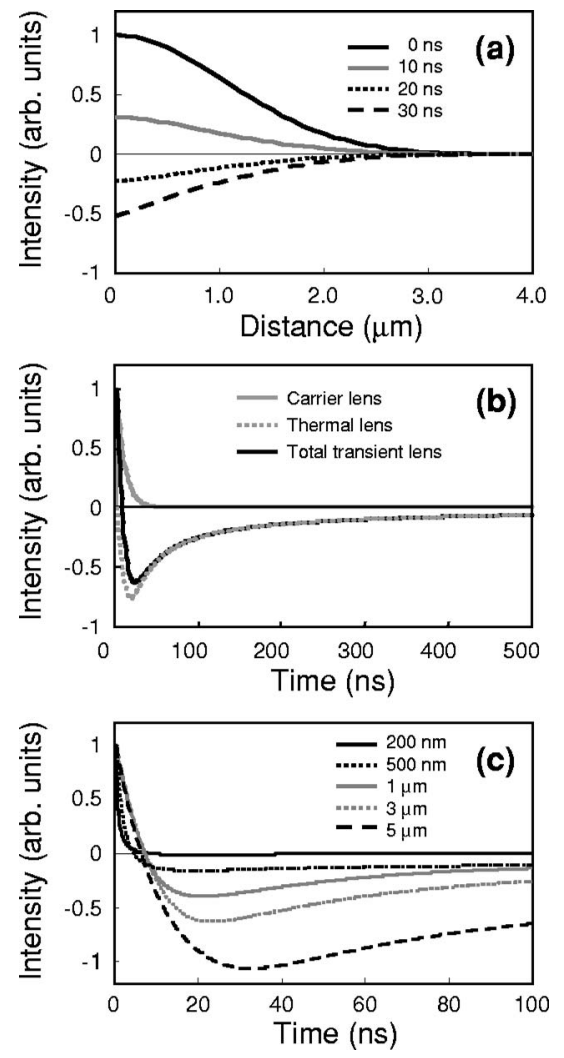

FIG. 3. Results of the numerical calculations. (a) Time-dependent special distribution of the transient lens. (b) Time profiles of the carrier lens, thermal lens, and total transient lens signals. (c) Spot size dependence of the time profiles of the transient lens signals.

Gaussian shaped excitation spot with $\phi=3 \mu \mathrm{m}$. Figure 3(b) shows the time profiles of the carrier lens, thermal lens, and total TL signals. This calculated time profile is similar to the experimentally obtained profile (Fig. 2). This fact suggests that our analysis and assignment of the TL signal is reasonable. Figure 3(c) presented the $\phi$ dependence of the TL time profiles. The time profile of the NSOM-TL with $200 \mathrm{~nm}$ aperture size is very difficult to experimentally detect, however we can estimate it by this calculation. Figure 3(c) shows that the contribution of the $\delta T$ becomes smaller with decreasing of $\phi$ and almost vanishes at $\phi=200 \mathrm{~nm}$. Thus, the NSOM-TL signal of our setup should be attributed to only the $\delta N$ contribution.

Figure 4 shows obtained (a) NSOM-PL and (b) NSOM-TL images with $3 \times 3 \mu \mathrm{m}$ scanning areas. We found a submicron scale spatial inhomogeneity in both NSOM-PL and NSOM-TL images. The spatial inhomogeneity of NSOM-PL is well known and interpreted to be the inhomogeneity of indium composition by the phase separation. ${ }^{3-16}$ The bright regions correspond to the high density region of the carrier localization centers, which act as the radiative recombination centers (RCs). On the other hand, the spatial inhomogeneity of NSOM-TL should denote the carrier distribution in the InGaN active layer because we already knew that the NSOM-TL signal is attributed to only the $\delta N$ [Fig. 3(c)]. We found the clear correlation between both PL and TL images. The bright regions in NSOM-PL, surrounded by the black dotted lines in Fig. 4, are corresponding to the bright regions in NSOM-PL. These regions denote the areas which have high RC density. Interesting regions are the dark regions in NSOM-PL surrounded by the white dotted lines in o AlP license or copyright; see http://apl.aip.org/apl/copyright.jsp 
(a)
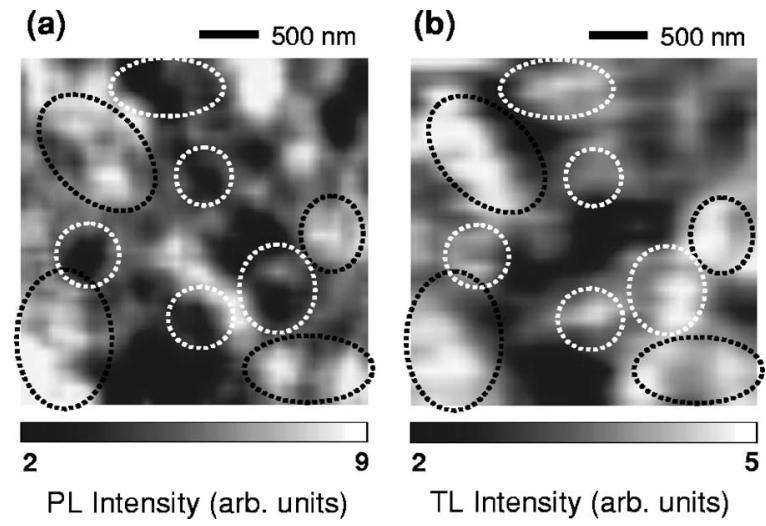

FIG. 4. (a) NSOM-photoluminescence (NSOM-PL) and (b) NSOM-TL images for $\mathrm{InGaN} / \mathrm{GaN} \mathrm{QW}$. The scanning area is $3 \times 3 \mu \mathrm{m}$. The regions surrounded by the black and white dotted lines are radiative and nonradiative recombination centers, respectively.

Fig. 4. These regions are corresponding to the bright regions in NSOM-PL. At these regions, the carrier density is high but emission is low. We consider that these regions denote the nonradiative recombination centers (NRCs), which had not so far been detected directly. The NRCs should be attributed to the point defects and/or threading dislocations, which are present all over the sample but are too small to detect by this technique. Their densities are distributed inhomogeneously and the high density areas are detected as NRC in Fig. 4(b).

There are other regions which have both dark PL and TL signal. These regions should be the high potential energy regions, which have low carrier density and low emission properties, since the generated carriers diffuse out from these regions. Previously, dark regions in NSOM-PL image have been considered to correspond to the NRC. However, the NRC and the high potential energy regions cannot by discriminated from each other by the conventional methods. Here we succeeded in such discrimination and the overall carrier diffusion and localization dynamics are schematized in Fig. 5. Similar schematics have already been reported by the time-resolved NSOM measurement. ${ }^{15,16}$ In this method, the carrier diffusion and localization dynamics were obtained by comparing the PL intensities and PL lifetime at each pixel of the NSOM image, and also by comparing the NSOM image with illumination mode and illumination-collection mode. The NSOM-TL presented here is a much easier tech-

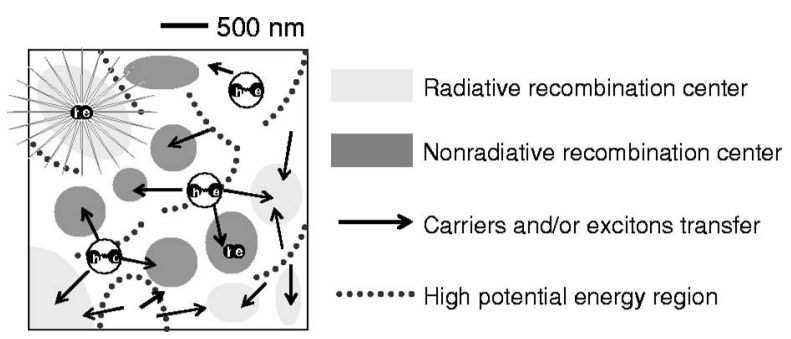

FIG. 5. Schematics of the diffusion and recombination carrier dynamics as observed in Fig. 4. nique with simple setup and easy operation, and it provides for direct observation of the NRC.

We conclude that the TR-M-TL and NSOM-TL are powerful techniques to observe the nonradiative carrier dynamics, which has been very difficult to detect by the conventional methods, in semiconductor materials. The NSOM-TL image gives the carrier density distribution in the active layer. By comparing NSOM-PL and NSOM-TL images, both the RC and NRC were unambiguously discriminated with submicrometer scales.

The authors would like to thank Professor Sg. Fujita and Professor M. Terazima (Kyoto University) for valuable suggestions and discussions. The authors also wish to thank G. Maltezos (Caltech) for helpful discussions and reviewing the manuscript. A part of this study was supported by AFOSR for their support under Contract No. FA9550-04-1-0413.

${ }^{1}$ S. Nakamura, T. Mukai, and M. Senoh, Appl. Phys. Lett. 64, 1687 (1994).

${ }^{2}$ S. Nakamura and G. Fasol, The Blue Laser Diode: GaN Based Light Emitting and Lasers (Springer, Berlin, 1997).

${ }^{3}$ T. Sugahara, M. Hao, T. Wang, D. Nakagawa, Y. Naoi, K. Nishino, and S. Sakai, Jpn. J. Appl. Phys., Part 2 37, L1195 (1998).

${ }^{4}$ S. Chichibu, K. Wada, and S. Nakamura, Appl. Phys. Lett. 71, 2346 (1997).

${ }^{5}$ F. Bertram, S. Srinvasan, L. Geng, F. A. Ponce, T. Riemann, and J. Christen, Appl. Phys. Lett. 80, 3524 (2002).

${ }^{6}$ D. K. Young, M. P. Mack, A. C. Abare, M. Hansen, L. A. Coldren, S. P. Denbaars, E. L. Hu, and D. Awschalom, Appl. Phys. Lett. 74, 2349 (1999).

${ }^{7}$ M. S. Jeong, J. Y. Kim, Y.-W. Kim, J. O. White, E.-K. Suh, C.-H. Hong, and H. J. Lee, Appl. Phys. Lett. 79, 976 (2001).

${ }^{8}$ M. S. Jeong, Y.-W. Kim, J. O. White, E.-K. Suh, M. G. Cheong, C. S. Kim, C.-H. Hong, and H. J. Lee, Appl. Phys. Lett. 79, 3440 (2001).

${ }^{9}$ A. Kaneta, T. Izumi, K. Okamoto, Y. Kawakami, Sg. Fujita, Y. Narita, T. Inoue, and T. Mukai, Jpn. J. Appl. Phys., Part 1 40, 110 (2001).

${ }^{10}$ H. Itoh, S. Watanabe, M. Goto, N. Yamada, M. Misra, A. Y. Kim, and S. A. Stockman, Jpn. J. Appl. Phys., Part 2 42, L1244 (2003).

${ }^{11}$ R. Micheletto, N. Yoshimatsu, A. Kaneta, Y. Kawakami, and Sg. Fujita, Appl. Surf. Sci. 229, 338 (2004).

${ }^{12}$ S. J. Chua, S. Tripathy, P. Chen, E. Takasuka, and M. Ueno, Physica E (Amsterdam) 25, 356 (2005).

${ }^{13}$ K. Okamoto, J. Choi, Y. Kawakami, M. Terazima, T. Mukai, and Sg. Fujita, Jpn. J. Appl. Phys., Part 1 43, 839 (2004).

${ }^{14}$ K. Okamoto, A. Kaneta, Y. Kawakami, Sg. Fujita, J. Choi, M. Terazima, and T. Mukai, J. Appl. Phys. 98, 064503 (2005).

${ }^{15}$ A. Kaneta, K. Okamoto, Y. Kawakami, Sg. Fujita, G. Marutsuki, Y. Narukawa, and T. Mukai, Appl. Phys. Lett. 81, 4353 (2002).

${ }^{16}$ A. Kaneta, T. Mutoh, Y. Kawakami, Sg. Fujita, G. Marutsuki, Y. Narukawa, and T. Mukai, Appl. Phys. Lett. 83, 3462 (2003).

${ }^{17}$ K. Okamoto, Y. Kawakami, Sg. Fujita, and M. Terazima, Anal. Sci. 17, s312 (2001).

${ }^{18}$ K. Okamoto, A. Kaneta, K. Inoue, Y. Kawakami, M. Terazima, T. Mukai, G. Shinomiya, and Sg. Fujita, Phys. Status Solidi B 228, 81 (2001).

${ }^{19}$ K. Okamoto, K. Inoue, Y. Kawakami, Sg. Fujita, M. Terazima, A. Tsujimura, and I. Kidoguchi, Rev. Sci. Instrum. 74, 575 (2003).

${ }^{20}$ K. Okamoto, Sg. Fujita, Y. Kawakami, and A. Scherer, Phys. Status Solidi B, 240, 368 (2003).

${ }^{21}$ M. Fujinami, K. Toya, and T. Sawada, Rev. Sci. Instrum. 74, 621 (2003).

${ }^{22}$ H. Haag, B. Hönerlage, O. Briot, and R. L. Aulombard, Phys. Rev. B 60, 11624, (1999).

${ }^{23}$ L. Siozade, S. Colard, M. Mihailovic, J. Leymarie, A. Vasson, N. Grandjean, M. Leroux, and J. Massies, Jpn. J. Appl. Phys., Part 1 39, 20 (2000). 